\title{
FOURTH - ORDER IMPROVED FINITE DIFFERENCE APPROACH TO PURE BENDING ANALYSIS OF LINE CONTINUUM.
}

\author{
O.M. Ibearugbulem ${ }^{1}$, J. C. Ezeh ${ }^{2}$, U.O. Okpara ${ }^{3}$ \\ ${ }^{1,2,3}$ Department of Civil Engineering, Federal University of Technology, Owerri, Nigeria.
}

\begin{abstract}
Analytical Methods provides exact solutions which have restrictions due to their inherent difficulties. On the other hand, Numerical methods provide approximate solution to governing differential equations. In this research, Improved Finite Difference Method (IFDM) using Polynomial series was used to develop a finite difference pattern. The patterns were applied in analyzing pure bending of line continuum with various boundary conditions. The results obtained from this numerical method were compared with those from exact method to check the accuracy of the solution. The results were found to be identical and very close to the exact results, with percentage difference ranging from $0-0.02 \%$. Hence, Improved Finite Difference Method provides simple and approximate solution that are close to the exact value for pure bending analysis of line continuum.
\end{abstract}

Key Words: Improved Finite Difference, Polynomial Series, Linear continuum, Numerical Methods...

\section{INTRODUCTION}

The behavior of flexural linear continuum was defined by formulating governing differential equations. These problems were solved by means of various analytical approaches to obtain exact solution (Chaje, 1974). Due to their inherent difficulties, such analytical and exact solutions have restrictions (Ibearugbulem et al., 20I3; Iyengar, 1988). In areas of considerable practical interest, they are either difficult or impossible to obtain. Hence, numerical methods are engaged to obtain approximate solutions (Ugural, 1999). Thus Finite Difference Method is regarded as a numerical method that has its merit due to its straight forward approach and minimum requirement on hardware.

\subsection{FINITE DIFFERENCE METHOD}

The Finite Difference Method is one of the most general numerical techniques. It refers to the process of replacing the partial derivatives by finite difference quotient and then obtaining solution of resulting system of algebraic equations (Yoo and Lee, 2011). In applying this method, the derivatives in the governing differential equation under consideration are replaced by differences at selected nodes. These nodes make the finite difference mesh.

The first order expansion gives the form

$f_{i}^{I}(x)=\frac{f_{i+1}-f_{i}}{\Delta x}$

Equation (1) is known as the First Forward Difference and
$f_{i}^{I}(x)=\frac{f_{i}-f_{i-1}}{\Delta x}$

Equation (2) is known as the First Backward Difference

Where $\Delta \mathrm{x}$ means grid spacing/ length of segments along member; i means station number.

Hence, the Central Difference is given as

$f_{i}^{I}(x)=\frac{f_{i+1}-f_{i-1}}{2 \Delta x}$

\section{POLYNOMIAL SERIES.}

Many numerical methods are based on Polynomial series expansion which is given as a function, $\mathrm{f}(\mathrm{x})$

$f_{(x)}=f_{(x i)}+\sum_{k=i}^{\infty} \frac{x-x_{i}}{k_{i}} f^{k}\left(x_{i}\right)$

Where $\mathrm{f}^{(\mathrm{k})}=\mathrm{k}^{\text {th }}$ derivative of the function.

Equation (4) can be re-written as

$$
\begin{gathered}
f(x)=f\left(x_{i}\right)+\frac{\left(x-x_{i}\right)}{1 !} f^{I}\left(x_{i}\right)+\frac{\left(x-x_{i}\right)^{2}}{2 !} f^{I I}\left(x_{i}\right)+ \\
\ldots \ldots .+\frac{\left(x-x_{i}\right)^{n}}{n !} f^{n}\left(x_{i}\right) \ldots \ldots \ldots \ldots \ldots \ldots \text { (5) }
\end{gathered}
$$

Applying equation (5) to expand the function $\mathrm{f}(\mathrm{x})$ at points $(\mathrm{x}+1)$ and $(\mathrm{x}-1)$ yields 


$$
\begin{aligned}
& f_{(x+1)}=f\left(x_{i}\right)+\frac{\Delta x}{1 !} f^{I}\left(x_{i}\right)+\frac{(\Delta x)^{2}}{2 !} f^{I I}\left(x_{i}\right)+ \\
& \frac{(\Delta x)^{3}}{3 !} f^{I I I}\left(x_{i}\right)+\frac{(\Delta x)^{4}}{4 !} f^{I V}\left(x_{i}\right)+\cdots \\
& +\frac{(\Delta x)^{n}}{n !} f^{(n)}\left(x_{i}\right) \\
& f_{(x-1)}=f\left(x_{i}\right)-\frac{\Delta x}{1 !} f^{I}\left(x_{i}\right)+\frac{(\Delta x)^{2}}{2 !} f^{I I}\left(x_{i}\right) \\
& -\frac{(\Delta x)^{3}}{3 !} f^{I I I}\left(x_{i}\right)+\frac{(\Delta x)^{4}}{4 !} f^{I V}\left(x_{i}\right)-\cdots \\
& +\frac{(-\Delta x)^{n}}{n !} f^{(n)}\left(x_{i}\right)
\end{aligned}
$$

\section{IMPROVED FINITE DIFFERENCE FORMULATION.}

For the derivation of improved derivatives, the polynomial series is applied once the grid parts are evenly spaced. Figure 1 schematically indicates the grid parts for central differences.

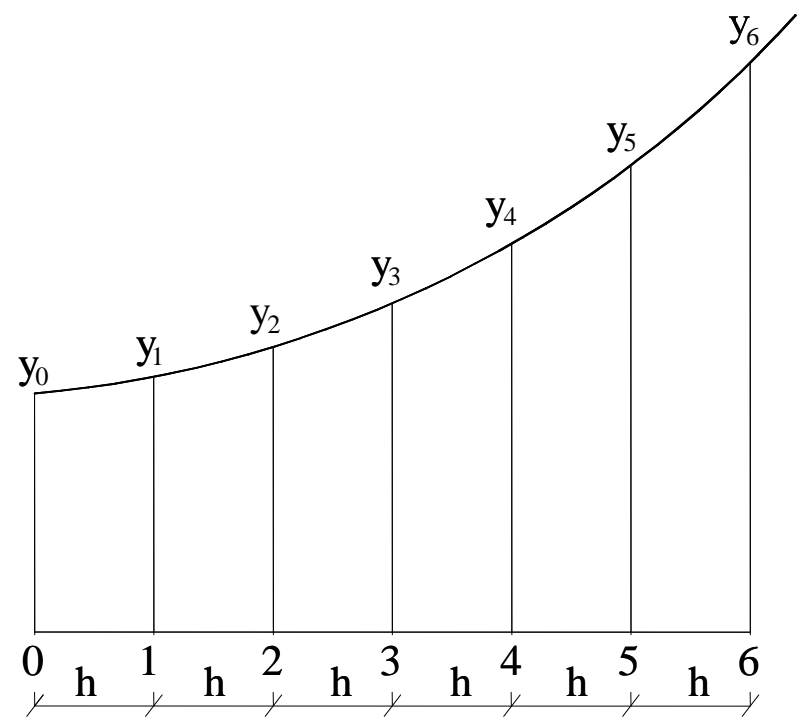

Figure 1: Computational grid for finite difference approximation

$\mathrm{h}$ is the grid spacing / segment length.

Taking $y_{3}$ as the pivotal point and expanding the function $y_{3}$ using the series and truncating at the fourth-order derivative $y^{\text {IV }}$ gave;

$$
\begin{aligned}
y_{4}=y_{3}+h y_{3}^{I} & +\left(\frac{h^{2}}{2}\right) y_{3}^{I I}+\left(\frac{h^{3}}{6}\right) y_{3}^{I I I} \\
& +\left(\frac{h^{4}}{24}\right) y_{3}^{I V} \ldots \ldots(8) \\
y_{2}=y_{3}-h y_{3}^{I} & +\left(\frac{h^{2}}{2}\right) y_{3}^{I I}-\left(\frac{h^{3}}{6}\right) y_{3}^{I I I} \\
& +\left(\frac{h^{4}}{24}\right) y_{3}^{I V} \ldots \ldots(9)
\end{aligned}
$$

Subtracting equations (9) from equation (8) gave:

$$
\begin{aligned}
& y_{4}-y_{2}=2 h y_{3}^{I}+\left(\frac{h^{3}}{3}\right) y_{3}^{I I I} . \text { That is } \\
& y_{3}^{I}=\frac{y_{4}}{2 h}-\frac{y_{2}}{2 h}-\left(\frac{h^{2}}{6}\right) y_{3}^{I I I} \ldots \ldots \ldots \ldots(10)
\end{aligned}
$$

Adding equations (8) and (9) gave:

$y_{4}+y_{2}=2 y_{3}+h^{2} y_{3}^{I I}+\frac{h^{4}}{12} y_{3}{ }^{I V}$. That is

$y_{3}^{I I}=\frac{1}{h^{2}}\left(y_{2}-2 y_{3}+y_{4}\right)-\left(\frac{h^{2}}{12}\right) y_{3}^{I V}$

Taking differences between nodes $\mathrm{y}_{3}$ and $\mathrm{y}_{5}$ and $\mathrm{y}_{3}$ and $\mathrm{y}_{1}$ equations (8) and (9) shall give:

$$
\begin{aligned}
y_{5}=y_{3}+2 h y_{3}^{I} & +\left(\frac{4 h^{2}}{2}\right) y_{3}^{I I}+\left(\frac{8 h^{3}}{6}\right) y_{3}^{I I I} \\
& +\left(\frac{16 h^{4}}{24}\right) y_{3}^{I V} \quad \ldots \\
y_{1}=y_{3}-2 h y_{3}^{I} & +\left(\frac{4 h^{2}}{2}\right) y_{3}^{I I}-\left(\frac{8 h^{3}}{6}\right) y_{3}^{I I I} \\
& +\left(\frac{16 h^{4}}{24}\right) y_{3}^{I V} \quad \ldots
\end{aligned}
$$

Subtracting equation (13) from equation (12) gives:

$y_{5}-y_{1}=4 h y_{3}^{I}+\left(\frac{8 h^{3}}{3}\right) y_{3}^{I I I}$

Adding equations (12) and (13) gives:

$y_{3}+y_{1}=2 y_{3}+4 h^{2} y_{3}^{I I}+\left(\frac{16 h^{4}}{12}\right) y_{3}^{I V}$

From equation (14), we have:

$y_{3}^{I}=\frac{1}{4 h}\left(y_{5}+y_{1}\right)-\left(\frac{2 h^{2}}{3}\right) y_{3}^{I I I}$

From equation (15), we have:

$y_{3}^{I I}=\frac{1}{4 h^{2}}\left(y_{5}+y_{1}-2 y_{3}\right)-\left(\frac{4 h^{2}}{12}\right) y_{3}^{I V}$

Subtracting equation (16) from equation (10) gave:

$0=\frac{y_{4}}{2 h}-\frac{y_{5}}{4 h}-\frac{y_{2}}{2 h}+\frac{y_{1}}{4 h}-\left(\frac{h^{2}}{6}\right) y_{3}^{I I I}+\left(\frac{2 h^{2}}{3}\right) y_{3}^{I I I}$

That is;

$0=3 \frac{y_{4}}{h}-3 \frac{y_{5}}{2 h}-3 \frac{y_{2}}{h}+3 \frac{y_{1}}{2 h}-h^{2} y_{3}^{I I I}+4 h^{2} y_{3}^{I I I}$ 
This implies;

$3 h^{2} y_{3}^{I I I}=\frac{-3 y_{1}}{2 h}+\frac{3 y_{2}}{h}-\frac{3 y_{4}}{h}+\frac{3 y_{5}}{2 h}$

$\therefore y_{3}^{I I I}=\frac{1}{2 h^{3}}\left(-y_{1}+2 y_{2}-2 y_{4}+y_{5}\right)$

Subtracting equation (17) from equation (11) gives:

$0=\frac{y_{2}}{h^{2}}-\frac{2 y_{3}}{h^{2}}+\frac{y_{4}}{h^{2}}-\frac{h^{2} y_{3}{ }^{I V}}{12}-\frac{y^{5}}{4 h^{2}}+\frac{2 y_{3}}{4 h^{2}}$

$$
+\left(\frac{4 h^{2}}{12}\right) y_{3}^{I V}
$$

That is;

$$
\begin{gathered}
0=12 y_{2}-24 y_{3}+12 y_{4}-h^{4} y_{3}^{I V}-3 y_{5}-3 y_{1}+6 y_{3} \\
+4 h^{4} y_{3}^{I V}
\end{gathered}
$$

This implies;

$3 h^{4} y_{3}^{I V}=3 y_{1}-12 y_{2}+18 y_{3}-12 y_{4}+3 y_{5}$

$\therefore y_{3}^{I V}=\frac{1}{h_{4}}\left(y_{1}-4 y_{2}+6 y_{3}-4 y_{4}+y_{5}\right)$.

Substituting equations (18) into equation (10) gives:

$y_{3}^{I}=\frac{y_{4}}{2 h}-\frac{y_{2}}{2 h}-\frac{h^{2}}{6}\left(\frac{1}{2 h^{3}}\left(-y_{1}+2 y_{2}-2 y_{4}+y_{5}\right)\right)$

That is;

$y_{3}^{I}=\frac{y_{4}}{2 h}-\frac{y_{2}}{2 h}+\frac{y_{1}}{12 h}-\frac{y_{2}}{6 h}+\frac{y_{4}}{6 h}-\frac{y_{5}}{12 h}$

$\therefore y_{3}^{I}=\frac{1}{12 h}\left(y_{1}-8 y_{2}+8 y_{4}-y_{5}\right)$

Equation (20) shows the first derivative of the improved finite difference.

Substituting equation (19) into equation (11) gives:

$y_{3}^{I I}=\frac{y_{2}}{h^{2}}-\frac{2 y_{3}}{h^{2}}+\frac{y_{4}}{h^{2}}$

$$
\begin{aligned}
& -\frac{h^{2}}{12}\left(1 / h^{4}\left(y_{1}-4 y_{2}+6 y_{3}-4 y_{4}\right.\right. \\
& \left.\left.+y_{5}\right)\right)
\end{aligned}
$$

That is;

$$
\begin{aligned}
y_{3}^{I I}=\frac{y_{2}}{h^{2}}-\frac{2 y_{3}}{h^{2}} & +\frac{y_{4}}{h^{2}}-\frac{y_{1}}{12 h^{2}}+\frac{y_{2}}{3 h^{2}}-\frac{y_{3}}{2 h^{2}}+\frac{y_{4}}{3 h^{2}} \\
& -\frac{y_{5}}{12 h^{2}}
\end{aligned}
$$$$
\therefore y_{3}^{I I}=\frac{1}{12 h^{2}}\left(-y_{1}+16 y_{2}-30 y_{3}+16 y_{4}\right.
$$

$$
\left.-y_{5}\right) \ldots . .(21)
$$

Equation (21) shows the second derivatives of the improved finite difference.

From equation (8), we can obtain:

$y_{4}^{I}=y_{3}^{I}+h y_{3}^{I I}+\frac{h^{2}}{2} y_{3}^{I I I}+\frac{h^{3}}{6} y_{3}^{I V}+\frac{h^{4}}{24} y_{3}^{V}$

From equation (9), we can obtain:

$y_{2}^{I}=y_{3}^{I}-h y_{3}^{I I}+\frac{h^{2}}{2} y_{3}^{I I I}-\frac{h^{3}}{2} y_{3}^{I V}+\frac{h^{4}}{24} y_{3}^{I V}$

Subtracting equation (23) from equation (22) gives:

$y_{4}^{1}-y_{2}^{1}=2 h y_{3}^{I I}+\frac{y^{I I}}{3} y_{3}^{I V}$ $\therefore y_{3}^{I I}=\frac{1}{2 h}\left(y_{4}^{I}-y_{2}^{I}\right)-\frac{h^{2}}{6} y_{3}^{I V}$

Adding equation (22) and equation (23) gives:

$y_{4}^{I}+y_{2}^{I}=2 y_{3}^{I}+h^{2} y_{3}^{I I I}+\frac{h^{4}}{12} y_{3}^{V}$
$\therefore y_{3}^{I I I}=\frac{1}{h^{2}}\left(y_{2}^{I}-2 y_{3}^{I}+y_{4}^{I}\right)-\frac{h^{2}}{12} y_{3}^{V}$

From equation (12) and equation (13), we have:

$$
\begin{aligned}
y_{5}^{I}=y_{3}^{I}+2 h y_{3}^{I I} & +\frac{4 h^{2}}{2} y_{3}^{I I I}+\frac{8 h^{3}}{6} y_{3}^{I V} \\
& +\frac{16 h^{4}}{24} y_{3}^{V} \\
y_{1}^{I}=y_{3}^{I}-2 h y_{3}^{I I} & +\frac{4 h^{2}}{2} y_{3}^{I I I}-\frac{8 h^{3}}{6} y_{3}^{I V} \\
& +\frac{16 h^{4}}{24} y_{3}^{V}
\end{aligned}
$$

Subtracting equation (27) from equation (26) gives:

$y_{5}^{I}-y_{1}^{I}=4 h y_{3}^{I I}+\frac{8 h^{3}}{3} y_{3}^{I V}$

Adding equation (26) and equation (27) gives:

$y_{5}^{I}+y_{1}^{I}=2 y_{3}^{I}+4 h y^{2} y_{3}^{I I I}+\frac{16 h^{4}}{12} y_{3}^{V}$

From equation (28), we can obtain:

$y_{3}^{I I}=\frac{1}{4 h}\left(y_{5}^{I}-y_{1}^{I}\right)-\frac{2 h^{2}}{3} y_{3}^{I V}$

From equation (29), we can obtain:

$y_{3}^{I I I}=\frac{1}{4 h^{2}}\left(y_{1}^{I}-2 y_{3}^{I}+y_{5}^{I}\right)-\frac{4 h^{2}}{12} y_{3}^{V}$.

Subtracting equation (30) from equation (24) gives:

$0=\frac{y_{4}{ }^{I}}{2 h}-\frac{y_{2}^{I}}{2 h}-\frac{h^{2}}{6} y_{3}^{I V}-\frac{y_{5}{ }^{I}}{4 h}+\frac{y_{1}^{I}}{4 h}+\frac{2 h^{2}}{3} y_{3}^{I V}$

That is;

$0=\frac{6 y_{4}^{I}}{h^{3}}-\frac{6 y_{2}^{I}}{h^{3}}-2 y_{3}^{I V}-\frac{3 y_{5}^{I}}{h^{3}}+\frac{3 y_{1}^{I}}{h^{3}}+8 y_{3}^{I V}$

This implies that

$6 y_{3}^{I V}=\frac{1}{h^{3}}\left(-3 y_{1}^{I}+6 y_{2}^{I}-6 y_{4}^{I}+3 y_{5}^{I}\right)$

$\therefore y_{3}^{I V}=\frac{1}{2 h^{3}}\left(-y_{1}^{I}+2 y_{2}^{I}-2 y_{4}^{I}+y_{5}^{I}\right)$

Subtracting equation (31) from equation (25) gives:

$0=\frac{y_{2}^{I}}{h^{2}}-\frac{2 y_{3}^{I}}{h^{2}}+\frac{y_{4}^{I}}{h^{2}}-\frac{h^{4}}{12} y_{3}^{V}-\frac{y_{1}^{I}}{4 h^{2}}+\frac{2 y_{3}^{I}}{4 h^{2}}-\frac{y_{5}^{I}}{4 h^{2}}$

$$
+\frac{4 h^{2}}{12} y_{3}^{V}
$$

That is

$$
\begin{aligned}
& h^{2} y_{3}^{V}=\frac{y_{1}^{I}}{h^{2}}-\frac{4 y_{2}^{I}}{h^{2}}+\frac{6 y_{5}^{I}}{h^{2}}-\frac{4 y_{4}^{I}}{h^{2}}+\frac{y_{5}^{I}}{h^{2}} \\
& \therefore y_{3}^{I}=\frac{1}{h^{4}}\left(y_{1}^{I}-4 y_{2}^{I}+6 y_{3}^{I}-4 y_{4}^{I}+y_{5}^{I}\right) \ldots
\end{aligned}
$$

Substituting equation (32) into equation (24), we have:

$y_{3}^{I I}=\frac{1}{2 h}\left(y_{4}^{I}-y_{2}^{I}\right)$

$$
-\frac{h^{2}}{6}\left(\frac{1}{2 h^{3}}\left(-y_{1}^{I}+2 y_{2}^{I}-2 y_{4}^{I}+y_{5}^{I}\right)\right)
$$


That is;

$=\frac{y_{4}^{I}}{2 h}-\frac{y_{2}^{I}}{2 h}+\frac{1}{12 h} y_{1}^{I}-\frac{1}{6 h} y_{2}^{I}+\frac{1}{6 h} y_{4}^{I}-\frac{1}{12 h} y_{5}^{I}$

$\therefore y_{3}^{I I}=\frac{1}{12 h}\left(y_{1}^{I}-8 y_{1}^{I}+8 y_{4}^{I}-y_{5}^{I}\right)$

Substituting equation (33) into equation (25), we have:

$y_{3}^{I I I}=\frac{1}{h^{2}}\left(y_{2}^{I}-2 y_{3}^{I}+y_{4}^{I}\right)$

$$
-\frac{h^{2}}{12}\left(\frac{1}{h^{4}}\left(y_{1}^{I}-4 y_{2}^{I}+6 y_{3}^{I}-4 y_{4}^{I}+y_{5}^{I}\right)\right)
$$

That is;

$=\frac{1}{h^{2}}\left(y_{2}^{I}-2 y_{3}^{I}+y_{4}^{I}\right)$

$$
-\frac{1}{12 h^{2}}\left(y_{1}^{I}-4 y_{2}^{I}+6 y_{3}^{I}-4 y_{4}^{I}+y_{5}^{I}\right)
$$

$\therefore y_{3}^{I I I}=\frac{1}{12 h^{2}}\left(y_{1}^{I}-16 y_{2}^{I}-30 y_{3}^{I}+16 y_{4}^{I}-y_{5}^{I}\right)$

Ignoring higher order term in equation (10) gives:

$y_{3}^{I}=\frac{1}{2 h}\left(y_{4}-y_{2}\right)$

Similarly;

$$
\begin{aligned}
y_{2}^{I} & =\frac{1}{2 h}\left(y_{3}-y_{1}\right) \\
y_{1}^{I} & =\frac{1}{2 h}\left(y_{2}-y_{2}\right) \\
y_{4}^{I} & =\frac{1}{2 h}\left(y_{5}-y_{3}\right) \\
y_{5}^{I} & =\frac{1}{2 h}\left(y_{6}-y_{4}\right)
\end{aligned}
$$

Substituting equations (36) to (34) into equation (35) gives:

$$
\begin{aligned}
y_{3}^{I I I}=\frac{1}{24 h^{3}}( & y_{2}+y_{0}+16 y_{3}-16 y_{1}-30 y_{4} \\
& \left.+30 y_{2}+16 y_{5}-16 y_{3}-y_{6}+y_{4}\right)
\end{aligned}
$$

That is;

$$
\begin{gathered}
=\frac{1}{24 h^{3}}\left(y_{0}-16 y_{1}+29 y_{2}-29 y_{4}+16 y_{5}-y_{6}\right) \\
\therefore \boldsymbol{y}_{3}^{I I I}=\frac{1}{\mathbf{2 4 h ^ { 3 }}}\left(\boldsymbol{y}_{0}-\mathbf{1 6} \boldsymbol{y}_{1}+29 y_{2}+\mathbf{0} y_{3}-29 y_{4}\right. \\
\left.\quad+\mathbf{1 6} \boldsymbol{y}_{5}-\boldsymbol{y}_{6}\right)
\end{gathered}
$$

Equation (41) shows the third derivatives of the improved finite difference.

From equation (22) and (23), we can obtain:

$y_{4}^{I I}=y_{3}^{I I}+h y_{3}^{I I I}+\frac{h^{2}}{2} y_{3}^{I V}+\frac{h^{3}}{6} y_{3}^{V}+\frac{h^{4}}{24} y_{3}^{V I}$

$y_{2}^{I I}=y_{3}^{I I}-h y_{3}^{I I I}+\frac{h^{2}}{2} y_{3}^{I V}-\frac{h^{3}}{6} y_{3}^{V}+\frac{h^{4}}{24} y_{3}^{V I}$

Adding equations (42) and (43) gives:
$y_{4}^{I I}+y_{2}^{I I}=2 y_{3}^{I I}+h^{2} y_{3}^{I V}+\frac{h^{4}}{12} y_{3}^{V I}$

This implies that

$y_{3}^{I V}=\frac{1}{h^{2}}\left(y_{2}^{I I}-2 y_{3}^{I I}+y_{4}^{I I}\right)-\frac{h^{2}}{12} y_{3}^{V I}$

From equations (26) and (27), we can obtain;

$$
\begin{gathered}
y_{5}^{I I}=y_{3}^{I I}+2 h y_{3}^{I I I}+\frac{4 h^{2}}{2} y_{3}^{I V}+\frac{8 h^{3}}{6} y_{3}^{V} \\
+\frac{16 h^{2}}{24} y_{3}^{V I} \\
y_{1}^{I I}=y_{3}^{I I}-2 h y_{3}^{I I I}+\frac{4 h^{2}}{2} y_{3}^{I V}-\frac{8 h^{3}}{6} y_{3}^{V} \\
+\frac{16 h^{4}}{24} y_{3}^{V I}
\end{gathered}
$$

Adding equations (45) and (46) gives:

$y_{5}^{I I}+y_{1}^{I I}=2 y_{3}^{I I}+4 h^{2} y_{3}^{I V}+\frac{16 h^{4}}{12} y_{3}^{V I}$

This implies that:

$y_{3}^{I V}=\frac{1}{4 h^{2}}\left(y_{1}^{I I}-2 y_{3}^{I I}+y_{5}^{I I}\right)-\frac{4 h^{2}}{12} y_{3}^{V I}$

Subtracting equation (47) from equation (44) gives:

$$
\begin{aligned}
\frac{y_{2}^{I I}}{h^{2}}-\frac{2 y_{3}^{I I}}{h^{2}}+\frac{y_{4}^{I I}}{h^{2}} & -\frac{h^{2}}{12} y_{3}^{V I}-\frac{y_{1}^{I I}}{4 h^{2}}+\frac{2 y_{3}^{I I}}{4 h^{2}}-\frac{y_{5}^{I I}}{4 h^{2}} \\
& +\frac{4 h^{2}}{12} y_{3}^{V I}=0
\end{aligned}
$$

This implies that:

$$
\begin{aligned}
& y_{3}^{V I}=-\frac{4 y_{2}^{I I}}{h^{4}}+\frac{6 y_{3}^{I I}}{h^{4}}-\frac{4 y_{4}^{I I}}{h^{4}}+\frac{y_{1}^{I I}}{h^{4}}+\frac{y_{5}^{I I}}{h^{4}} \\
& \therefore y_{3}^{V I}=\frac{1}{h^{4}}\left(y_{1}^{I I}-4 y_{2}^{I I}+6 y_{3}^{I I}-4 y_{4}^{I I}+y_{5}^{I I}\right) . .
\end{aligned}
$$

Substituting equation (48) into equation (47) gives:

$$
\begin{aligned}
y_{3}^{I V}=\frac{1}{4 h^{2}}\left(y_{1}^{I I}\right. & \left.-2 y_{3}^{I I}+y_{5}^{I I}\right) \\
& -\frac{4 h^{2}}{12}\left(\frac{1}{h^{4}}\left(\begin{array}{c}
y_{1}^{I I}-4 y_{2}^{I I}+6 y_{3}^{I I} \\
-4 y_{4}^{I I}+y_{5}^{I I}
\end{array}\right)\right)
\end{aligned}
$$

That is;

$=\frac{y_{1}^{I I}}{4 h^{2}}-\frac{2 y_{3}^{I I}}{4 h^{2}}+\frac{y_{5}^{I I}}{4 h^{2}}-\frac{y_{1}^{I I}}{3 h^{2}}+\frac{4 y_{2}^{I I}}{3 h^{2}}-\frac{6 y_{3}^{I I}}{3 h^{2}}+\frac{4 y_{4}^{I I}}{3 h^{2}}-\frac{y_{5}^{I I}}{3 h^{2}}$

That is;

$=\frac{-y_{1}^{I I}}{12 h^{2}}+\frac{4 y_{2}^{I I}}{3 h^{2}}-\frac{5 y_{3}^{I I}}{2 h^{2}}+\frac{4 y_{4}^{I I}}{3 h^{2}}-\frac{y_{5}^{I I}}{12 h^{2}}$ 
$\therefore y_{3}^{I V}=\frac{1}{12 h^{2}}\left(-y_{1}^{I I}+16 y_{2}^{I I}-30 y_{3}^{I I}+16 y_{4}^{I I}\right.$

$$
\left.-y_{5}^{I I}\right)
$$

Ignoring higher order term in equation (11) gives:

$y_{3}^{I I}=\frac{1}{h^{2}}\left(y_{2}-2 y_{3}+y_{4}\right)$

Similarly;

$$
\begin{aligned}
& y_{2}^{I I}=\frac{1}{h^{2}}\left(y_{1}-2 y_{2}+y_{3}\right) \ldots \ldots \ldots( \\
& y_{1}^{I I}=\frac{1}{h^{2}}\left(y_{0}-2 y_{1}+y_{2}\right) \ldots \ldots \ldots( \\
& y_{4}^{I I}=\frac{1}{h^{2}}\left(y_{3}-2 y_{4}+y_{5}\right) \ldots \ldots \ldots(53 \\
& y_{5}^{I I}=\frac{1}{h^{2}}\left(y_{4}-2 y_{5}+y_{6}\right) \ldots \ldots \ldots(54
\end{aligned}
$$

Substituting equations (50) to (54) into equation (49) gives:

$$
\begin{aligned}
y_{3}^{I V}=\frac{1}{12 h^{4}} & \left(-y_{0}+2 y_{1}-y_{2}+16 y_{1}-32 y_{2}\right. \\
& +16 y_{3}-30 y_{2}+60 y_{3}-30 y_{4}+16 y_{3} \\
& \left.-32 y_{4}+16 y_{5}-y_{4}+2 y_{5}-y_{6}\right)
\end{aligned}
$$

That is;

$$
\begin{aligned}
& =\frac{1}{12 h^{4}}\left(-y_{0}+18 y_{1}-63 y_{2}+92 y_{3}-63 y_{4}+18 y_{5}\right. \\
& \left.-y_{6}\right) \\
& \therefore y_{3}^{I V}=\frac{1}{12 h^{4}}\left(-y_{0}+18 y_{1}-63 y_{2}+92 y_{3}-63 y_{4}\right. \\
& \left.+18 y_{5}-y_{6}\right) \ldots(55)
\end{aligned}
$$

\begin{tabular}{|c|c|}
\hline$y_{3}^{n}$ & COEFFICIENTS \\
\hline $12 h^{2} y_{3}^{I I}$ & $-1-16--30-16--1$ \\
\hline $12 h y_{3}^{I}$ & $1--8-0-8--1$ \\
\hline $24 h^{3} y_{3}^{I I I}$ & $-1-16-29-0--29-16--1$ \\
\hline $12 h^{4} y_{3}^{I V}$ & $-1-18--63-92--63-18--1$ \\
\hline
\end{tabular}

Equation (55) shows the fourth derivative of the improved finite difference.

The coefficients of the corresponding function values in the improved finite difference expressions of the first-order, second-order, third-order and fourth-order derivatives as given in equations (20), (21), (41) and (55) are used to develop finite difference patterns and are schematically given on table 1.
Table -1: Schematic representation of Higher order Finite Difference Expression.

\section{BASIC EQUATION FOR PURE BENDING.}

The fourth - order differential equation of a bent line continuum was given by Ugural (Ugural, 1999) as:

$$
E I \frac{d^{4} w}{d x^{4}}=P
$$

Where $\mathrm{w}$ is the deflection and $\mathrm{P}$ is the applied uniformly distributed load per meter length of the continuum.

\section{LINEAR CONTINUUM WITH VARIOUS BOUNDARY CONDITIONS.}

Three line continua with different boundary conditions were studied in this work namely: Pin - Roller supports $(\mathrm{P}-\mathrm{R})$, Clamped - Clamped supports $(\mathrm{C}-\mathrm{C})$ and Clamped - Roller supports $(\mathrm{C}-\mathrm{R})$. Their boundary conditions are shown in table 2. Also, the line continua were studied using 3 different cases namely:

Case 1: Dividing the linear continuum into 4 equal lengths

Case 2: Dividing the linear continuum into 6 equal lengths

Case 3: Dividing the linear continuum into 8 equal lengths 
Table -2: Line continua and their boundary conditions.

\begin{tabular}{|c|c|}
\hline Linear Continuum & Boundary Conditions \\
\hline $\begin{array}{l}\text { Pin } \quad-\quad \text { Roller Linear } \\
\text { Continuum }(\mathrm{P}-\mathrm{R})\end{array}$ & $\begin{array}{c}w_{(0)}=0, \frac{d^{2} w_{(0)}}{d x^{2}}=w_{(0)}^{I I}=0 ; \\
w_{(l)}=0, \frac{d w_{(L)}}{x d^{2}}=w_{(L)}^{I I}=0 .\end{array}$ \\
\hline $\begin{array}{l}\mid \\
\text { Clamped - Clamped } \\
\text { Linear Continuum }(\mathrm{C}-\mathrm{C})\end{array}$ & $\begin{array}{l}w_{(0)}=0, \frac{d w_{(0)}}{d w}=w_{(0)}^{I}=0 ; \\
w_{(L)}=0, \frac{d^{2} w_{(L)}}{d x^{2}}=w_{(L)}^{I}=0\end{array}$ \\
\hline $\begin{array}{l} \\
\text { Clamped - Roller Linear } \\
\text { Continuum (C-R) }\end{array}$ & $\begin{array}{l}w_{(0)}=0, \frac{d w_{(0)}}{d w}=w_{(0)}^{I}=0 ; \\
w_{(L)}=0, \frac{d w_{(L)}}{d x}=w_{(L)}^{I}=0 .\end{array}$ \\
\hline
\end{tabular}

\section{APPLICATION AND RESULT OF IMPROVED FINITE DIFFERENCE ANALYSIS}

The derived coefficients and patterns are applied to the governing equation at each node using the 3 different cases with the appropriate boundary conditions being satisfied to generate the required matrix equations, from which the deflections at each node were determined. The results of the pure bending analysis are presented on table 3. Exact result obtained from previous analysis is also shown in the table for comparison.

Table -3a: Result data for case 1 and exact values

\begin{tabular}{|r|c|c|}
\hline $\begin{array}{r}\text { Linear } \\
\text { continuum }\end{array}$ & Exact result & $\begin{array}{c}\text { Case 1 } \\
(\mathrm{n}=3)\end{array}$ \\
\hline $\mathrm{P}-\mathrm{R}$ & $\frac{5 P L^{4}}{384 E I}$ & $\frac{0.013048 P L^{4}}{E I}$ \\
\hline $\mathrm{C}-\mathrm{C}$ & $\frac{P L^{4}}{384 E I}$ & $\frac{0.003348 P L^{4}}{E I}$ \\
\hline $\mathrm{C}-\mathrm{R}$ & $\frac{2 P L^{4}}{384 E I}$ & $\frac{0.005896 P L^{4}}{E I}$ \\
\hline & $3.2 \%$ Diff $)$ \\
\hline
\end{tabular}

Table -3b: Result data for case 2 and exact values

\begin{tabular}{|r|c|c|}
\hline $\begin{array}{r}\text { Linear } \\
\text { continuum }\end{array}$ & Exact result & $\begin{array}{c}\text { Case } 2 \\
(\mathrm{n}=5)\end{array}$ \\
\hline P-R & $\frac{5 P L^{4}}{384 E I}$ & $\frac{0.013026 P L^{4}}{E I}$ \\
\hline C-C & $\frac{P L^{4}}{384 E I}$ & $\frac{0.04 \% \text { Diff })}{(12.2 \% \text { Diff })}$ \\
\hline
\end{tabular}

\begin{tabular}{|c|c|c|}
\hline C-R & $2 P L^{4}$ & $0.005499 P L^{4}$ \\
\hline & $\overline{384 E I}$ & $\begin{array}{c}E L \\
(5.6 \% \text { Diff })\end{array}$ \\
\hline
\end{tabular}

Table -3c: Result data for case 3 and exact values

\begin{tabular}{|c|c|c|}
\hline $\begin{array}{r}\text { Linear } \\
\text { continuum }\end{array}$ & Exact result & $\begin{array}{c}\text { Case } 3 \\
(\mathrm{n}=7)\end{array}$ \\
\hline \multirow[t]{2}{*}{ P-R } & $5 P L^{4}$ & $0.013021 P L^{4}$ \\
\hline & $\overline{384 E I}$ & $\begin{array}{r}E I \\
(0 \% \text { Diff })\end{array}$ \\
\hline \multirow[t]{2}{*}{$\mathrm{C}-\mathrm{C}$} & $P L^{4}$ & $0.002779 P L^{4}$ \\
\hline & $\overline{384 E I}$ & $\begin{array}{c}E I \\
\text { (6.7\% diff) }\end{array}$ \\
\hline \multirow[t]{2}{*}{ C-R } & $2 P L^{4}$ & $0.005371 P L^{4}$ \\
\hline & $\overline{384 E I}$ & $\begin{array}{c}E I \\
(3.13 \% \text { Diff })\end{array}$ \\
\hline
\end{tabular}

\section{CONCLUSIONS}

The result for the pure bending analysis of line continua of three boundary conditions using Improved Finite Difference are presented on table 3 . It can be seen from the analysis that the results from P-R boundary condition are virtually the same as exact results. Results emerging from C-C boundary condition shows a percentage difference ranging from $6.7 \%$ $-28.6 \%$ while that of C-R boundary condition ranges from $3.13 \%-13.2 \%$ from the exact result.

Also, these results suggest that as the number of nodes (or division) increases, the more accurate the result becomes. Finally, the results from the pattern developed from this improved finite difference analysis are effective and is recommended for use in structural engineering.

\section{REFERENCES}

[1]. Alexander Chajes (1974). Structural Stability Theory New Jersey. Prentice - Hall, Inc.

[2]. Awele, M., Ayodele, J.C., and Osaisai, F.E. (2003). Numerical Methods for Scientists and Engineers. Ibadan. Beaver Publications.

[3]. Ibearugbulem, O. M., Ettu, L. O., Ezeh, J. C. and Anya, U. C. (2013). New Shape function for

Analysis of Line continuum by Direct Variational Calculus. International Journal of Engineering \& Science, Vol. 2, issue 3; Pp. 66 - 71, ISBN 2319 1805.

[4]. Goodwine, B. (2010). Engineering Differential Equations: Theory and Applications. New York: Springer Science + Business Media, LLC2011.

[5]. Szilard, R. (2004). Theories and Application of Plate Analysis. New Jersey: John Wiley and Sons Inc.

[6]. Ugural, A.C (1999). Stress in Plates and Shells $\left(2^{\text {nd }}\right.$ Ed.).Singapore: McGraw-Hill. 
[7]. Yoo, H.C. and Lee, C.S. (2011). Stability of Structures.

Principles and Applications. USA:

Butterworth- Heinemann. Elsevier Inc. 